\title{
SHEFEX - A First Aerodynamic Post-Flight Analysis
}

\author{
Tarik Barth and Thino Eggers \\ DLR, Institute of Design Aerodynamics, Lilienthalplatz 7, 38108 Braunschweig, Germany
}

\begin{abstract}
Summary
The given paper summarises the status of the post-flight analysis of the SHarp Edge Flight EXperiment (SHEFEX). It focuses on the comparison of numerical and experimental surface pressures as well as the assessment of the free stream vector applying the flight mechanic description of the Digital Miniature Attitude Reference System (DMARS) platform. The results point out that the extraction of angles of attack and sideslip is much more demanding than expected.
\end{abstract}

\section{Introduction}

Hypersonic systems are complex, difficult to design and expensive to build due to a lack of physical understanding on the involved flow regimes and a lack of data for design. The strategic future tool which will enable a dramatic reduction in the design and development time required for new vehicles is the computational or "virtual" vehicle design and qualification. But this approach is based on mathematical models which require verification and validation to increase their credibility. The improvement of the physical modelling requires good data, acquired in ground facilities and in flight [3].

The objective of the SHEFEX experiment was the investigation of a facetted Thermal Protection System (TPS) concept and the assessment of the potential of sharp edged configurations applying the three point strategy: numerical analysis - ground based facilities - flight experiment. The motivation is neither to perform a re-entry experiment nor to fly at the thermal boundary of modern high temperature materials but to prove in flight that the temperature peaks at the edges of the ceramic-composite panels are lower than those predicted based on a radiation equilibrium hypothesis. The SHEFEX forebody should have as many as possible facetted panels and it should represent as many as possible configuration details of space vehicles, like concave and convex chamfers and a sharp unswept leading edge, see Fig. 1(a). Now, after the flight an enormous amount of scientific data is available and a direct comparison of the flight data with numerical post flight studies and on-ground data is possible.

\section{Description of the Experiment}

The SHEFEX launcher is a two-stage solid propellant sounding rocket system. The launch vehicle consisted of a Brazilian S30 motor as first stage and an Improved 
Orion motor as second stage. Between the facetted SHEFEX experiment and the second stage were two cylindrical modules which housed the recovery system, the main electronics, the data acquisition devices, the power supply, and the cold gas system (Fig. 2). The vehicle reached an apogee of $211 \mathrm{~km}$. The total flight time was 550 seconds, comprising 45 seconds of experimental time for the atmospheric reentry between $90 \mathrm{~km}$ and $14 \mathrm{~km}$. The first atmospheric effects on the acceleration sensors could be observed at $80 \mathrm{~km}$. Here the pitch and yaw angles $(\psi$ and $\Theta$ ) started to oscillate and unfortunately the roll rate started to increase, see Fig. 3(a). The vehicle finally achieved a stable flight attitude with a decreasing ellipse-shaped precession around the flight vector, see Fig. 3(b). The flight data enable a detailed flight mechanic description of the complete flight. Data of 59 sensors distributed on the surface of the forebody like pressure transducers, thermocouples, and heat flux sensors are available for an analysis [2].

\section{Assessment of the Flow Condition}

One requirement for the numerical post-flight analysis is the availability of the free stream condition, namely, the Mach number $M_{\infty}$, angle of attack $\alpha$ and sideslip angle $\beta$. In order to obtain the Mach number or the Reynolds number the 1976 standard atmosphere [1] is applied. The angles of attack and sideslip have to be determined based on the flight vector provided by the DMARS platform. The DMARS flight vector is given in the platform coordinate system. Therefore, e.g. the pitch angle is zero during vertical ascent, $90^{\circ}$ in horizontal position and so on. The sideslip angle is the lateral deviation from the flight path (Fig. 4). As shown in Fig. 3(a) the re-entry configuration performed a very complex damped coning motion. The pitch and yaw angles oscillate around mean values which are indicated by the dash-dotted lines in the Fig. 3(b). Estimates for the mean values are $164.5^{\circ}$ for the pitching motion and $3^{\circ}$ for the yawing motion.

Now, $\alpha$ and $\beta$ are the angles between the longitudinal axis of the vehicle and the flight path, which is not directly known. But, considering Fig. 4, they correspond to the deviation from the mean values for pitch and yaw angle. The roll angle $\phi$ may be directly taken from the DMARS data.

The flight velocities during the atmospheric descent varied in the region of $1700 \mathrm{~m} / \mathrm{s}$. The Mach number, see Fig. 5(a), is relatively constant at a value of approx. 5.6 from $100 \mathrm{~km}$ down to $50 \mathrm{~km}$. Then the Mach number increases up to its maximum value 6.2 at $26 \mathrm{~km}$. The Reynolds number, see Fig. 5(b), increases up to $10 \cdot 10^{6}$ at payload separation. Therefore, a careful comparison of the pressure distributions, temperatures and the heat flux rates from laminar and turbulent Navier-Stokes calculations may allow to detect the altitude where boundary layer transition appears.

The actual flow condition and the corresponding experimental values may be obtained applying the procedure given in Fig. 6(a). The post-flight analysis at first required the exact adjustment of the time scales of Radar-, DMARS-, and experimenttime. The Radar data are used to extract the altitude and corresponding time. Applying this time values of pitch-, yaw- and roll angles as well as velocity are obtained 
from the DMARS data while the measured surface values are contained in the experimental database.

\section{Selected Results of the Post-Flight Analysis}

To compare the numerical with the experimental data Navier-Stokes calculations are performed using the TAU-CODE. Dependent on the altitude the boundary layer state is assumed to be laminar $(>40 \mathrm{~km})$ or turbulent $(<30 \mathrm{~km})$. Between 30 and $40 \mathrm{~km}$ both types are investigated. The wall is always defined to behave radiation adiabatic $(\epsilon=0.83)$. The comparisons of experimental and numerical pressure distributions are done along a line on the lower surface, see Fig. 6(b).

For the numerical calculation the velocity vector is only defined by the angles of attack and sideslip. The roll angle can not be taken into account. Therefore, an orientation $(\psi, \Theta$ and $\phi)$ and a grid generation of the vehicle have to be carried out for any time step in order to respect the correct flight position. Alternatively, the influence of roll angle may be included using a transformation of the angles of attack and sideslip. On account of the fact that the orientations and grid generations are much time consuming and error-prone the transformation is favoured. Consequently, the transformed values for the angles of attack $\alpha^{\prime}$ and sideslip $\beta^{\prime}$ which already include the effect of the roll angle are defined by equations (1) and (2).

$$
\begin{aligned}
& \alpha^{\prime}=\cos (\phi) \cdot \alpha+\sin (\phi) \cdot \beta \\
& \beta^{\prime}=\cos (\phi) \cdot \beta-\sin (\phi) \cdot \alpha
\end{aligned}
$$

Tab. 1 illustrates the influence of the roll angle on the angles of attack and sideslip. In case that the roll angle is zero, the transformed angle of attack $\alpha^{\prime}$ is identical to the angle of attack $\alpha$. If now an additional roll angle of $90^{\circ}$ is introduced at constant angle of attack $\alpha^{\prime}$ is identical to the sideslip angle $\beta$. The increase of the roll angle to $180^{\circ}$ ends to $\alpha^{\prime}=-\alpha$. A comparison of a result obtained on a rotated vehicle with the one obtained by application of the transformed angles and one additional application using transformed angles prove the procedure (Fig. 7).

With respect to save calculation time it is proposed to compute only half of the SHEFEX forebody. That is possible when the influence of the sideslip is marginal and a symmetric flow field may be assumed. For this purpose the influence of the sideslip angle is here investigated. The results point out, that a sideslip angle of less than $3^{\circ}$ has only minor influence and the half configuration may be used. In case of larger values the complete forebody has to be considered because the characteristic of the pressure distribution is changed (see Fig. 8 as example).

For the numerical calculation of the re-entry the following altitudes are selected: $19.0 \mathrm{~km}, 27.4 \mathrm{~km}, 33.8 \mathrm{~km}$, and $55.7 \mathrm{~km}$. The comparison of the pressure distributions for these altitudes are presented in Fig. 9. The agreement is for $19.0 \mathrm{~km}$ and $27.4 \mathrm{~km}$ reasonably good. Unfortunately, this promising agreement does not hold for the two considered higher altitudes. If the altitude reaches more than $55 \mathrm{~km}$, the influence of the measurement uncertainty $(\approx \pm 50 \mathrm{~Pa})$ has to be taken into account. 
For the altitude of $33.8 \mathrm{~km}$ a comparison of laminar and turbulent pressures with the experimental ones suggests that at this altitude the boundary layer may be turbulent. However even for the turbulent flow case large discrepancies in the forward and backward region of the body are observed. Especially in the region of the first pressure sensor the accuracy has to be much better, because here the flow is almost two dimensional and the pressure value has to be comparable with the oblique shock relations. Here the experimental pressures taken $50 \mathrm{~ms}$ later are much closer to the calculation but the agreement is still not promising. The clear influence of flight time on the measured pressure distribution points out that the very fast changes of the flight vector are one obvious problem. Therefore, possible explanations for the large discrepancies between experiment and simulation are the procedure to define the angle of attack and the sensitive adjustment of the experimental time scales (compare [4]).

In order to investigate in depth the discrepancies observed at high altitude, two flow conditions ( $39 \mathrm{~km}$ and $55 \mathrm{~km}$ ) with large offset from the experimental pressures are considered using the oblique shock relations given by equations (3) and (4).

$$
\begin{gathered}
\tan \vartheta=2 \cdot \cot \sigma \cdot\left(\frac{M_{1}^{2} \cdot \sin ^{2} \sigma-1}{M_{1}{ }^{2} \cdot(\kappa+\cos 2 \sigma)+2}\right) \\
\frac{p_{2}}{p_{1}}=\frac{2 \cdot \kappa \cdot M_{1 n}{ }^{2}-(\kappa-1)}{\kappa+1} \text { with } M_{1 n}{ }^{2}=M_{1} \cdot \sin \sigma
\end{gathered}
$$

The Fig. 10(a) shows that for both altitudes the offset is large and the downstream behaviour of the pressure distribution is not well captured for the assumed angles of attack (see section 3). The aim is to establish an analytic angle of attack $\alpha_{\text {shock }}$ using the oblique shock relations. In Fig. 10(b) is presented that the flow conditions are not fully two dimensional. Therefore, in a first step the numerical pressure directly at the leading edge is extracted which is obtained using the angle of attack defined in section 3). In oder to include three dimensional effects, a parallel translation of the frontward pressure distribution in a way that which agrees well with the first pressure sensor is being performed in the second step. Finally, a new hypothetic pressure $p_{2}$ may be extracted at the leading edge which agree with the pressure after the shock. Applying the known geometric deflection angle $\vartheta$ and the oblique shock relation within an iteration a new angle of attack may be found. The new angles $\left(\alpha_{\text {shock }}=3.27^{\circ}\right.$ for $39.1 \mathrm{~km}$ and $\alpha_{\text {shock }}=-5.30^{\circ}$ for $55.7 \mathrm{~km}$ ) allow a much better agreement with the measured values. Additional, this procedure shows for $55.7 \mathrm{~km}$ that the discrepancies are not caused by the measurement uncertainty, but rather may be traced back to uncertainty on the velocity vector direction.

\section{Conclusions}

An strategy to reduce the flight data of the SHEFEX experiment in order to establish an aerodynamic data basic has been presented and discussed. The results of the 
paper point out that the extraction of angles of attack and sideslip is much more demanding than expected and that relatively straightforward experiments like SHEFEX with an enormous amount of available scientific data and the quite demanding problems of the flight analysis are an essential source to acquire knowledge in the physics of hypersonic flight and the development of more complex vehicles. The presented scheme of work enables to develop the SHEFEX aerodynamic data basic. For further experiments it will be recommended to include an aerodynamic air data system, which allows a direct determination of $\alpha$ and $\beta$.

\section{References}

[1] Digital Dutch: The 1976 Standard Atmosphere. http: //digitaldutch. com.

[2] Gülhan, A.; Requardt, G.; Siebe, F.; Koch, U.; Esser, B.: Instrumentation and In-Flight Data of the SHEFEX Flight Experiment. Proceedings of the 1st International ARA Days on Atmospheric Re-Entry Systems, Missions and Vehicles, Arcachon, France, June 2006

[3] Longo, J.M.A.: Aerothermodynamics A critical review at DLR. Aerosp. Sci. Technol. 7 (2003) 429-438.

[4] Turner, J.; Hörschgen, M.; Stamminger, A.; Turner, P.: SHEFEX Hypersonic Reentry Flight Experiment, Vehicle and Subsystem Design, Flight Performance and Prospects. AIAA Paper 2006-8115, 2006.

Table 1: Transformed angles of attack $\alpha^{\prime}$ and sideslip $\beta^{\prime}$

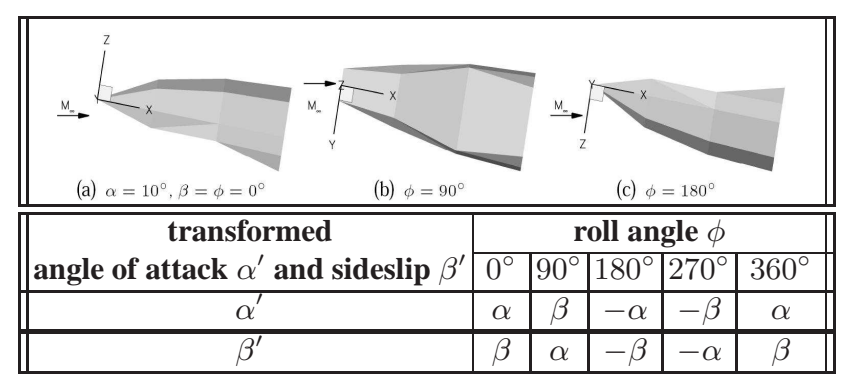

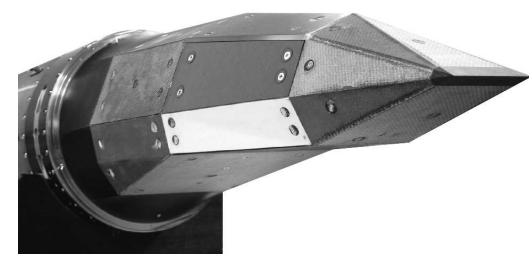

(a) Forebody

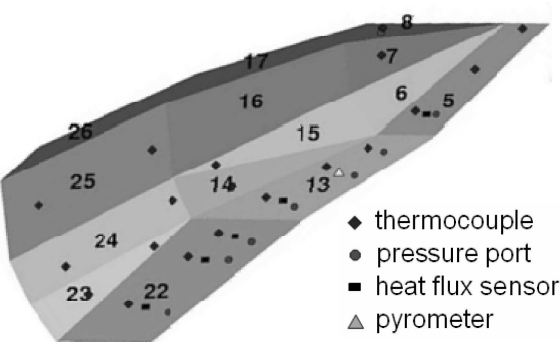

(b) Sensors

Figure 1: The SHEFEX Experiment 


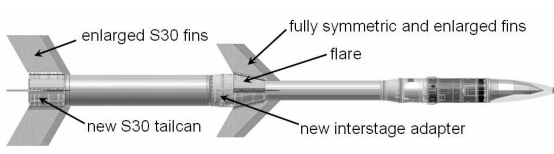

(a) Launcher

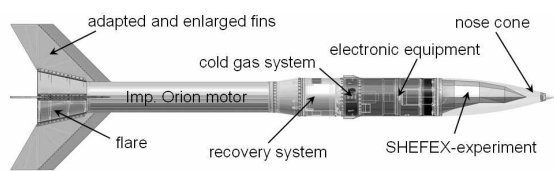

(b) Re-entry configuration

Figure 2: SHEFEX launch and re-entry configuration

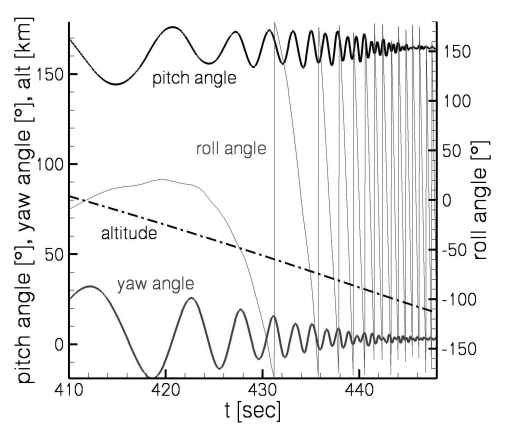

(a) Re-entry

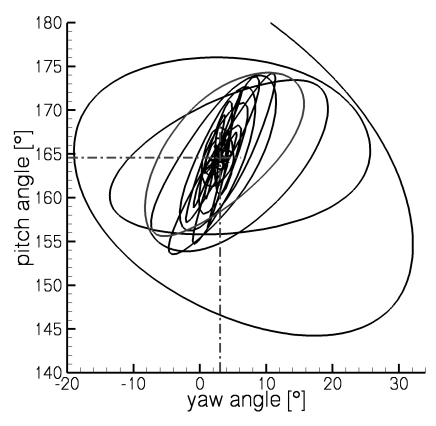

(b) Pitch and yaw with average

Figure 3: Re-entry description based on DMARS data

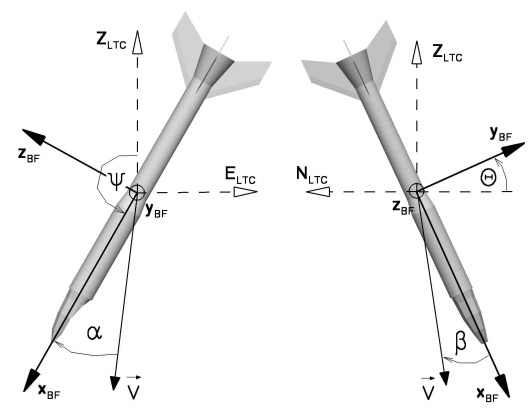

$\begin{array}{ll}\text { BF } & \text { Body Frame } \\ \text { LTC } & \text { Local Tangential } \\ & \text { Coordinate System } \\ \psi & \text { pitch angle } \\ \Theta & \text { yaw angle } \\ \alpha & \text { angle of attack } \\ \beta & \text { sideslipe angle }\end{array}$

Figure 4: Definition of pitch, yaw, attack, and sideslip angles

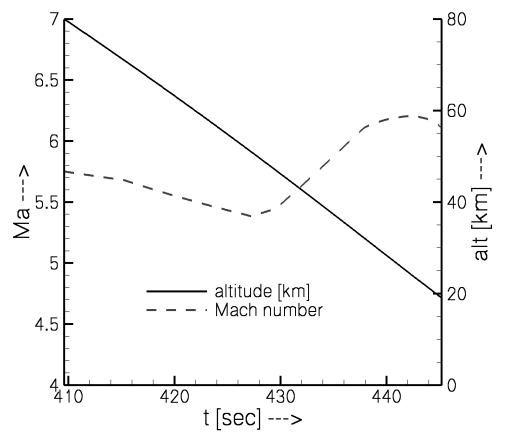

(a) Mach number, altitude

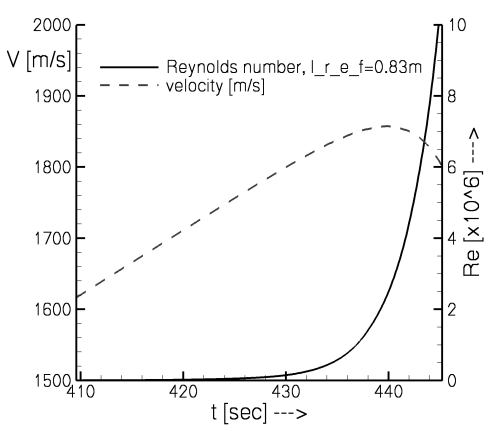

(b) Reynolds number, velocity

Figure 5: Flow conditions during atmospheric re-entry 


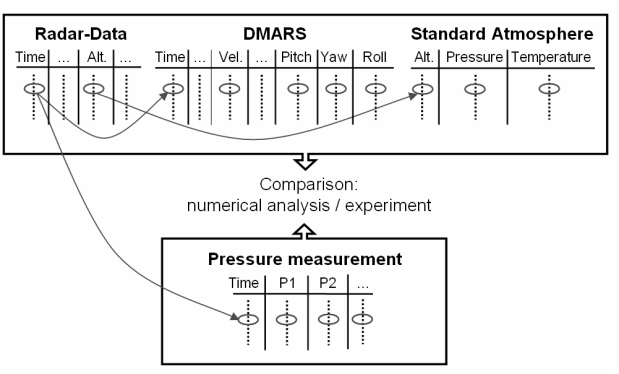

(a) Extraction of flight data

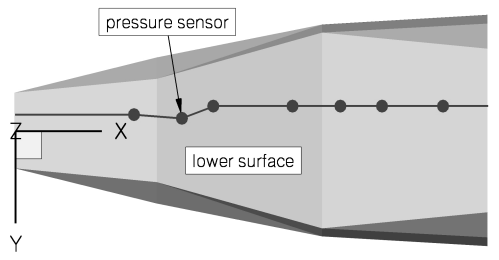

(b) Pressure sensors

Figure 6: Flight analysis
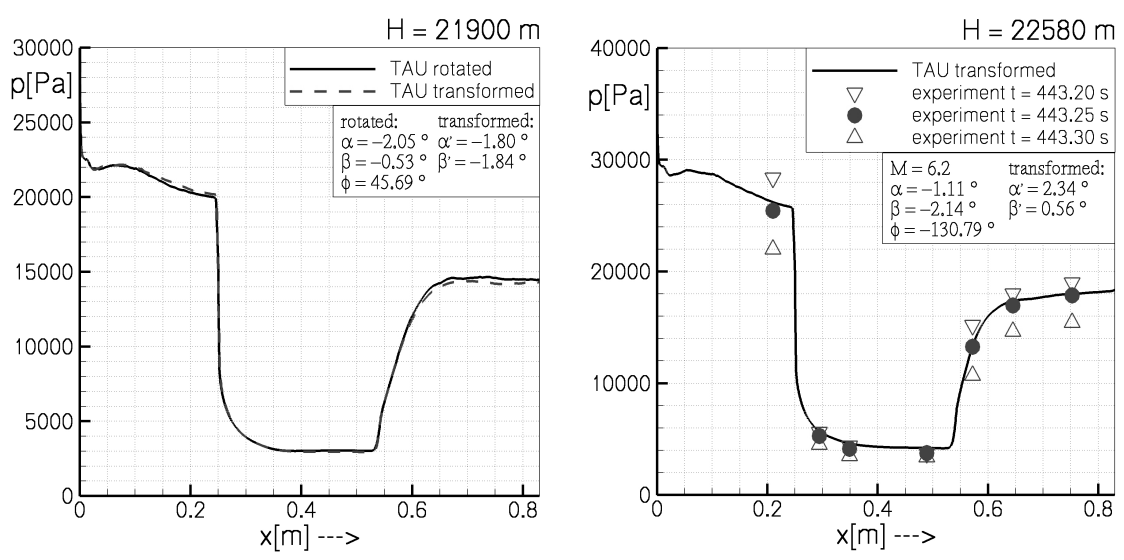

Figure 7: Comparison: rotated grid / transformed angles of attack and sideslip
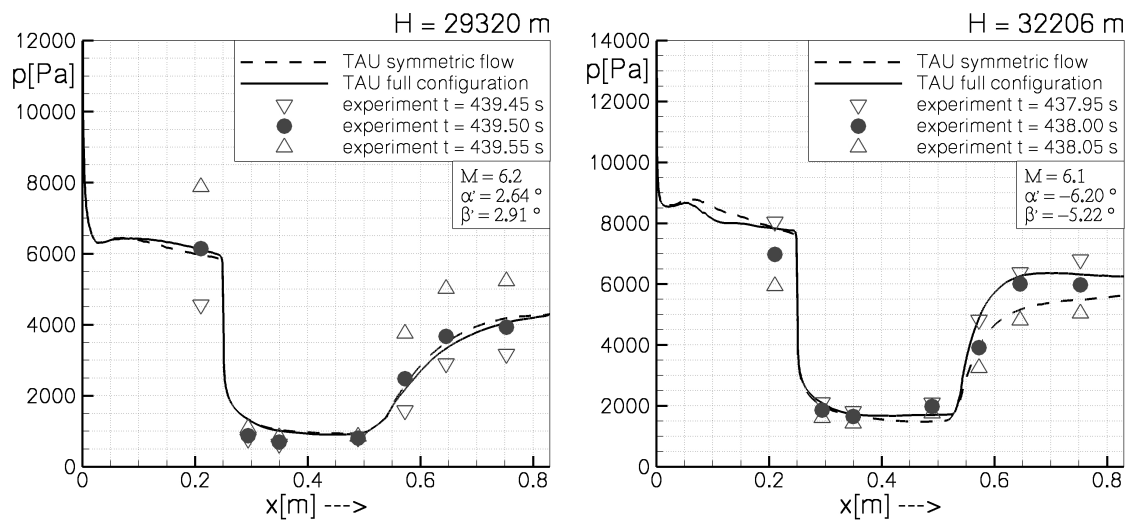

Figure 8: Numerical and experimental pressure distributions, influence of the sideslip angle 

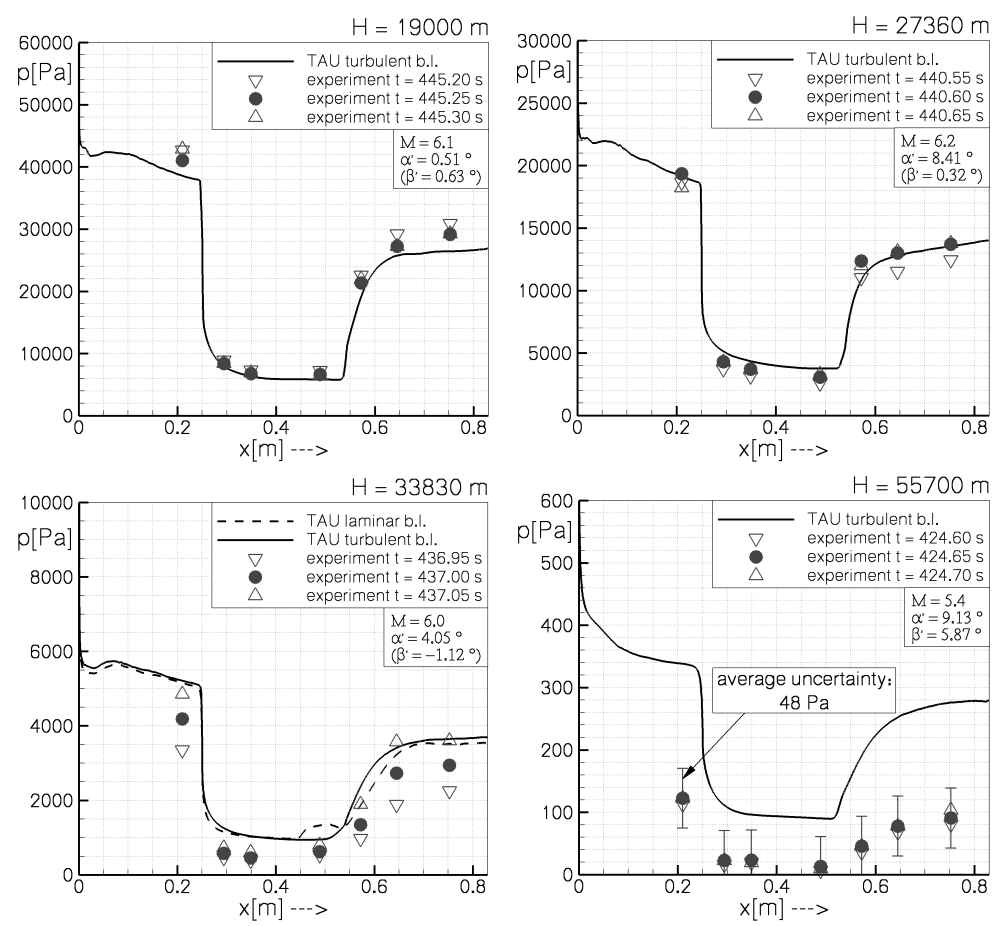

Figure 9: Numerical and experimental pressure distributions
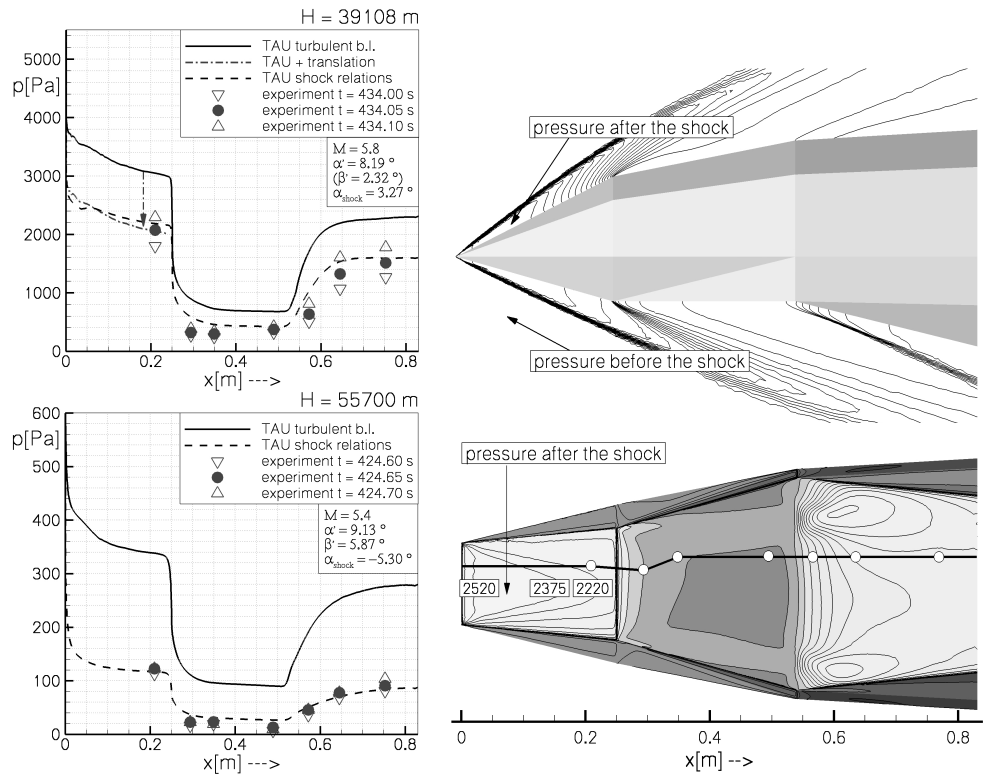

(a) Analysed altitudes

(b) Pressure gradients $(39 \mathrm{~km})$

Figure 10: Recalculation of the angle of attack based on the oblique shock relations 